\title{
Supplementary File 1
}

306 -

G. theta

$74------------------P K R Y K E S L E T R P S D D T L D S Y R--N I R P V C V V$

Chrysochromulina 138

C. owczarzaki

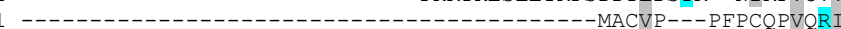

O. cincta

S. rosetta

L. anatina

C. gigas

L. gigantea

A. queenslandica

A. aurita

C. intestinalis

$\mathrm{N}$. vectensis

A. millepora

S. purpuratus

O. bimaculoides

D. citri

T. cancriformis

C. teleta

A. pisum

H. sapiens

B. floridae

F. occidentalis

C. aquilonaris

I. scapularis

A. geniculata

C. sculpturatus

S. olivacea

T. castaneum

S. maritima

E. danica

R. prolixus

$P$. humanus

C. pallens

Z. nevadensis

T. commodus

8 - - - - - - - - DPHAWPEV----------PSAAEHLP PDCFRSVHCAPMRSVPRC MDQVASHISQQIERGRLRLPGV--------------SSPVVNCRQLGV-ATQPIPRY ------MSETPFRDCLGGPGTP-----VPQPELNNGTGHYDGGDTHNYNE-ITGNVPCY $------M A T--A E V P S D G---------R K P H E K K E S G Y Y T S Q M F D Y F F-D T E D I P C I$

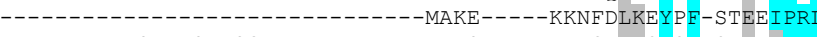
------MATSVERSPESG----------EDAYS-----TIRGELKQYSE-QTEPVPRI

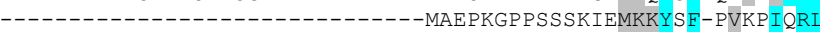

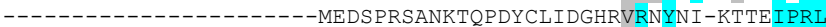
------------------------MAAVSDLYTDLDGHLVRKY SL-DVIQVPRI

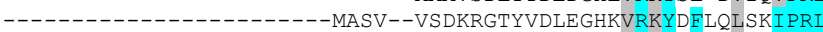
------------------------MESGEISS PAKK I PGLKYYTE-IEGKVPIC ------MAATAAEAVASG-----SGEP--REEAGALGPAWDESQLRSYSE-PTRPIPRI -------------------------MAGAEGWS SAQLRQYSE-PLETIPRI (1) 作 -

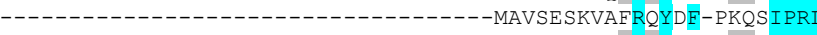
作 -_------------------------MKMAQNNIEWNENQLRKYDF-ILEDI PRI ------MRRRAAPH I LRGPPVESSTVR--TGTKMAOKRGYDASOLRKYDI Q QLEEI PRI

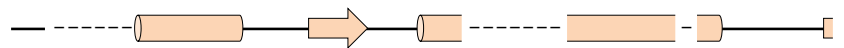

P. brassicae

E. huxleyi

B. natans

G. theta

4 DV---AADVVDAGDAAIQADRPVVVDGLAS---------RAGDWTVAK-LLDAIGDVQV 321 PFGDGGQATLDTLARYVEADQPVVLTGADIVDV-.-.----ERWSDNAKVEALLGDKAV

103 PWG------SPLLDQFLEAHLPIVIRGSQLIKRGGSTGESKESKWTPGH-IQSMLAAKK

Chrysochromulina 172 PFG-----SKAARALLEQRKPVILTHHTLVGG------AASKWTLDY-LCQQMHGLPC

C. owczarzaki 43 RAT------DDAARLALRSKTPFVLLDSSIAAP-_-----AVERWDLEF-LKEHLGMSN-

O. cincta 48 DFN------DPVALNLIKEQVPVILKGADLVSS-------A-MKWDLDY-LSTHIGGGRF

S. rosetta 21 RVC------DPKLLDFIREQRPIVITDSKLVES-------A-RHWDLEY-MEKHMGDCKF

L. anatina

C. gigas

L. gigantea

A. queenslandica

A. aurita

C. intestinalis

N. vectensis

A. millepora

S. purpuratus

O. bimaculoides

D. citri

T. cancriformis

C. teleta

A. pisum

H. sapiens

B. floridae

F. occidentalis

C. aquilonaris

I. scapularis

A. geniculata

C. sculpturatus

S. olivacea

T. castaneum

S. maritima

E. danica

R. prolixus

$P$. humanus

C. pallens

Z. nevadensis

T. commodus

40 NAST----QSSKVDQFIASERPVLMTNTDLVKT------A-LHWDLNY-LEQHLGDGDF

24 NID------NPETEKRIAAGLPVVITDSGLVRS---

29 CWT------EATADHLMTHEEPVVLTDTDLVQS-------A-LHWDLSY-LEQNMGPAKH

37 NFN-----DPEAAELISSQKPVVLTGTNLIEP-------A-LHWNLEY-LRDNIGDGEN

28 SIQ-----DPETYQLIKDSKPVVITDTNLVTS-------A-SKWDLEY-LNKNLGDGLF

30 HYQ-----DPKATEIIKNECPVVLTDSDIISS------A-MKWDIEY-LRENIGDGD

35 EYD------DPNVESI IANEEPVLLVNSNIISS-------A-LKWDLDY-LKKNLGAGSF

41 DVS------DPKVSEYVRDGKPVVITGTNLVET-------A-LKWDLDY-LEKNLGSGKF

23 PYD-----DPQAQRLISAEQPVVLTGSDLVDS------A-LHWNLDY-LSDNMANTQF

32 SFD-----DPRVDEYIGDMKPVLITGSNLIQS------A-MKWDLEY-ISEHMPNSNL

30 DAC------DPEALNLISKQFPVLLRNTRLIDS-------A-LHWDIEY-LERHAGSSDF

30 SCK-----DPRLNDVIAREEPVVITDCNLASS------A-SHWSLEY-LSSNIGNGTF

23 QYD-----DPKVDSLLSNNKPVLIKGSKLVSQ------V-LKWDLDY-LAEHMNS ICC

46 SQS-----DPRAEELIENEEPVVLTDTNLVYP-------A-LKWDLEY-LQENIGNGDF

26 SCT-----DPEADRLISEEKPVVLTDTHLIDS------A-LKWDLGY-LRANLGGGLC

27 NCM------DPQVDELIKSKKPVVLTGSNLVGA-------A-SKWDLDY-LEKNMGNGDF

26 SCH-----DPEAQRRIFAMQPVVLTDTNLVTS------A-LKWDLDY-LESNIGGGDY

31 SHT-----DPEADRLIANMMPVVLTDTGLVAP------A-LKWDLDY-LSEHLGEGSC

-A-LKWDLDY-LEANIGDGVF

22 SCL-----DPNADALIENMKPVVLTDTNLVSS-------A-LKWNLDY-LQKNLGDGSF

35 SAS-----DPLASQLIAEEKPVVLTDTNLCDT------A-LKWDLDY-LAQHMGSERY

27 HYK-----DPKIDEYIKENKPVVITESNIVKP------AVQRWSLEY-LERNLGHSGH

24 SIF-----DPEADKLISEERPIVLTDSNLIRP-------A-LKWDLEY-LESHLGPGNY

35 EYS------DARVDEFIAKDRPVVITGTCLVAP-------A-AKWTLEY-LAHNIGQGEF

28 KVS-----DPKVEELIAARKPVI IVDSDLVAP-------S-TKWDLNY-LQEHMGSTNC

28 RFD-----DPKVLELILQNKPVVILGSDLVKS------T-EKWDLEY-LEKNMGDSDF

29 DCN------DPNVDILISSNRPVVITGANLVNT-------A-NKWNLEY-LEKNMGNSDF

26 SVK-----DPKVDEFIADNKPVIITDTRLVAS-------A-ERWDLDY-LEQNMGNGD

51 HAS-----DPRVDELISENKPVVITGTDLVSS------A-MLWDLEY-LERNMGNGDY 


\begin{tabular}{|c|c|c|}
\hline sicae & & $----Q$ QSARF LYFKADRAGDTS---------- \\
\hline xleyi & 372 & -----GRRFRYWANDDPGARTAPGGGGGGGGG \\
\hline natans & 156 & YESP-- - - - - - - - - - - - - - - - - - - - - - SQ \\
\hline eta & 62 & ------------------------- SNFF $\mathrm{Y}$ WDEEKNAGQ ------------ \\
\hline ochromulina & 218 & $------------------------S$ \\
\hline owczarz & 88 & 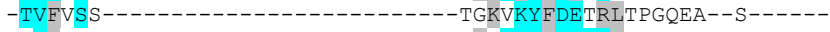 \\
\hline icta & 93 & EADRHALVQPQSSSNPQSQSVQPEPLHIFKYFDEKKLVALQD-------. \\
\hline etta & 6 & ------------------------- ERVFMFSDEEKNLG ----------- \\
\hline & & ------------------ NDKE LYHD \\
\hline & 69 & 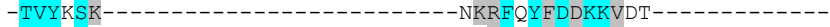 \\
\hline antea & 2 & 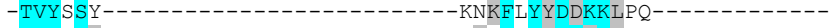 \\
\hline ndica & 74 & 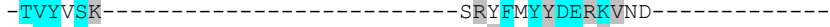 \\
\hline & 82 & $-----------------------n$ \\
\hline nalis & 73 & --------------------1 \\
\hline & 75 & -------------------1 \\
\hline & 80 & ------------------- \\
\hline & 86 & ----------------- \\
\hline & 68 & -----------------1 \\
\hline & 77 & ------------------1 \\
\hline & 75 & 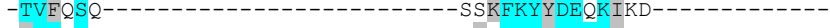 \\
\hline & 7 & 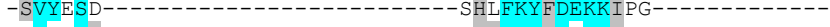 \\
\hline & 68 & ------------------- \\
\hline & 9 & ------------------- \\
\hline & 7 & ------------------ \\
\hline & 72 & ------------------- \\
\hline & 71. & 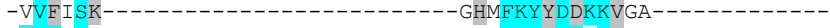 \\
\hline & 76 & 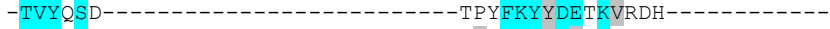 \\
\hline & 67 & $------------------------\mathrm{R}$ \\
\hline & 6 & $-------------------------\mathrm{N}$ \\
\hline & 80 & -----------------1 \\
\hline & & -------------------1 \\
\hline & 6 & 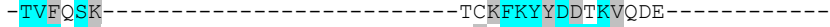 \\
\hline & 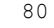 & -------------------1 \\
\hline & 73 & ------------------ \\
\hline anus & 7 & KVNQLLS-------- \\
\hline & 7 & - ----------------- \\
\hline & & - \\
\hline commodus & & \\
\hline
\end{tabular}

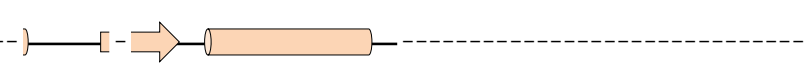

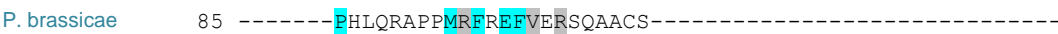

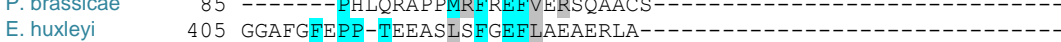

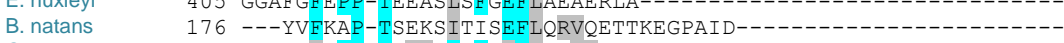

G. theta 82 -- YEYTPP-TSKIQMNFHDFVETIRRP-.--

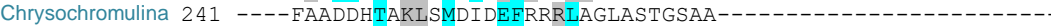

C. owczarzaki 111 ------HHDVRKLTMRFAEFLECMAVAARQTERFAPRPQSSCNEPTTSTQRDSCCQPTA

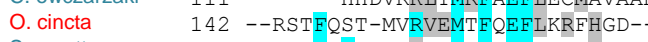

S. rosetta $\quad 86$--NYKFTPT-ATKRSMTFQEFASEFREA-

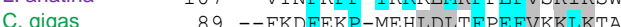

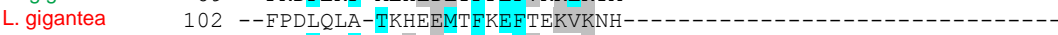

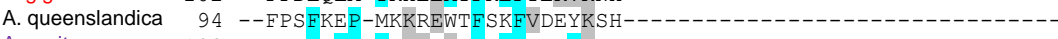

A. aurita $\quad 102-$ KYVNFQPP-TQKLSLTFYEFYELLRSW-

C. intestinalis 93 --CKSFVTP-SERHHVTFLQFLQMLKRC----

N. vectensis $\quad 96---$ PHFRPP-TVRIDMKFEQFYKQVNKF-----

A. millepora $101---$ PYFIPP-TQRIHMKFEEFCSRVRNL--

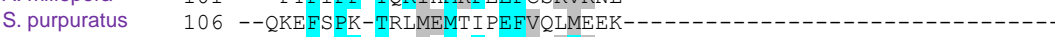

O. bimaculoides $88--O K D F V P P-T I O M O M S F S E F V E K L K E A-$

D. citri $100--$ STLEPITTRWSSMTAQEFSTRIKSW--

T. cancriformis $95--$ CPGFVPS-ITKLEIPFSNFAARLRQW----

C. teleta $\quad 95--H K D E R P E-M R R K E M K F N D F V A L M Q N P$

A. pisum $88--$ NMTEKPI-SRPSPMIFSEFAKKIKDW---

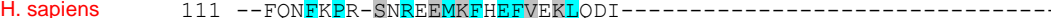

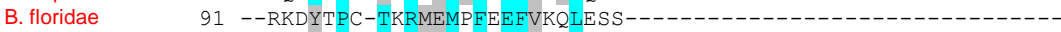

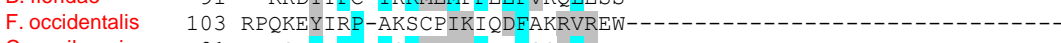

C. aquilonaris $\quad 91--R S K F I P P-T Q R I D L K F P V F S Q R L K E W-$

I. scapularis $\quad 97$-RLTDFRAP-TLRHEWKIGTFAQRLRSA-

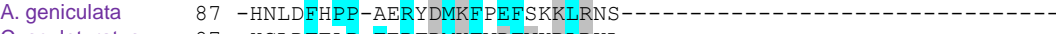

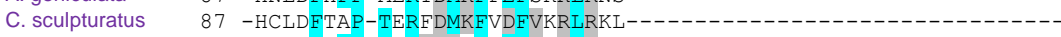

S. olivacea 100 -YKTNFVPP-TRRVDLTFPEFVKKLREW---

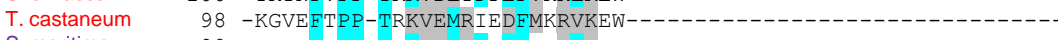

980 RTADVP-TRRIDMKEPEFAVKTRNT- -

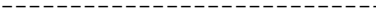

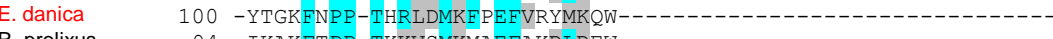

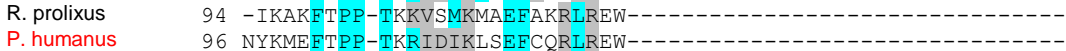

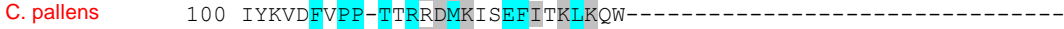

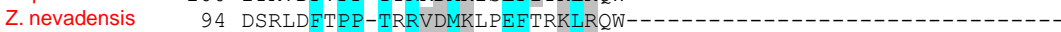

T. commodus 119 ESKAEFTPP-TRRVDMKLPEFSQRLKQW- 


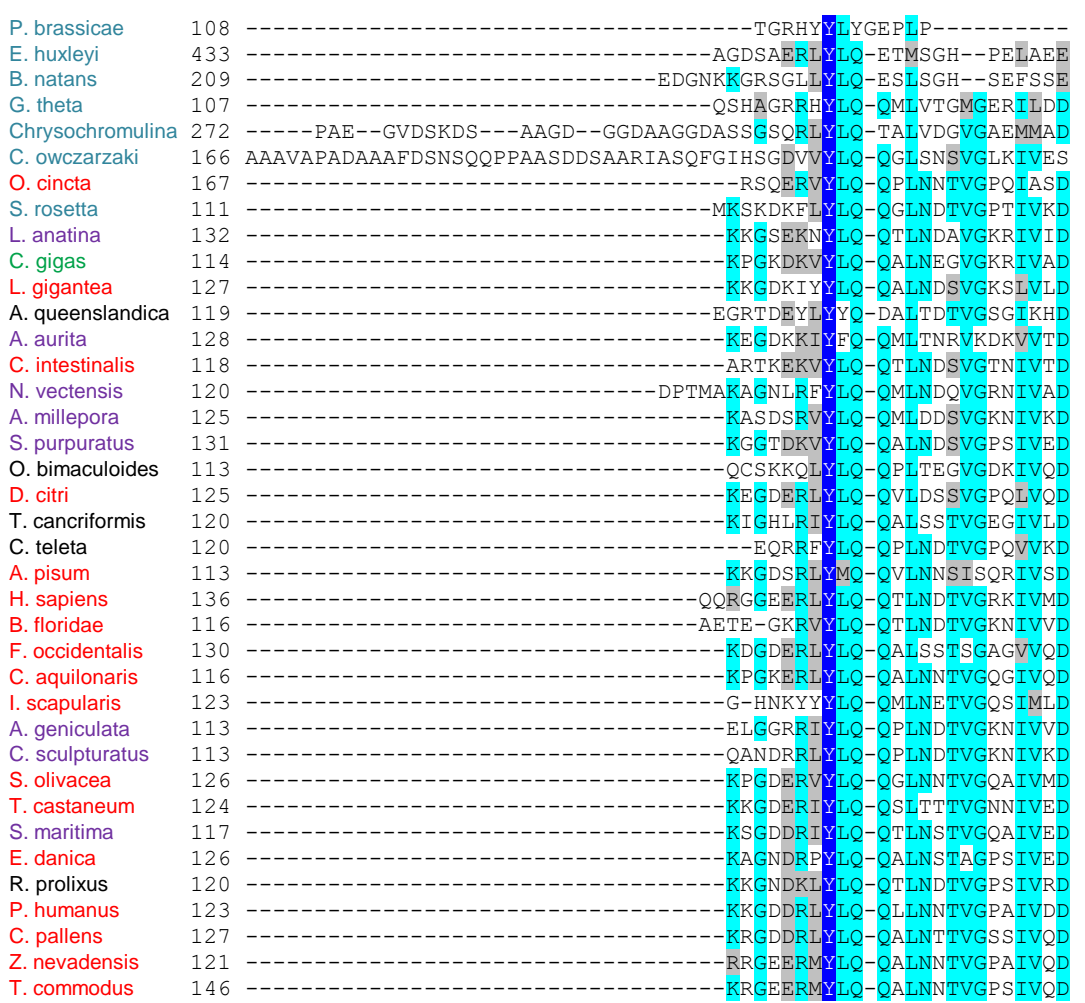

\begin{tabular}{|c|c|c|c|}
\hline P. brassicae & 121 & SALWRSLPDMERVLGSTAQLTSSLLWMALDATC & MCHGLLLQM \\
\hline E. huxleyi & 456 & RSWNWPFLLQNSNRH-RWGLPDSNELFIGMQGAE & RKQLLLFP \\
\hline B. natans & 236 & ASWNWNWLLRIVQKY-GWGTPDRNILLIGMQGAT & \\
\hline G. theta & 132 & FKHVDWGTVYGWKÃQL-GFGDLTTNLLLVGTTGHV & EQHNFFCQLQC \\
\hline Chrysochromulina & 319 & FRAFDWEGVLATQQRL-GWGSLSSNLLLVGQKGNT & EQQNLFAQLHG \\
\hline C. owczarzaki & 225 & FLQFNWKLLAELQRDA-NWSDLTTNLLLVSMAGAV & EQENLFAQVRRA \\
\hline O. cincta & 190 & FIQFNWSWAAEHKREF-NWGPLTSNLLLIGQKGNI & EQQNLFCPLRGR \\
\hline S. rosetta & 136 & FVGFNWTWLTDVVKAL-NWGHLTTNMLFVSMPPHLV & EQENFFAQVRGT \\
\hline L. anatina & 156 & FLGFNWDWINKQQNGN-NWGPLTANLLLIGQEGNY & EQHNLFAQVQGC \\
\hline C. gigas & 138 & FLGFNWSWVTEQQKKN-KFGALTSNLLLIGMEGNV & DEQENFFAQIRGYK \\
\hline L. gigantea & 151 & FIKFNWSWATEQQKKY-KWGALTSNLLLVGMDGVI & DEQQNFFAQIGGFKRFLLFD \\
\hline A. queenslandica & 144 & FVHFNWHWVSERKKRY-NWGQLTSNLLLISQPGNI & DEQHNFFCQVRGLKRCLLFA \\
\hline A. aurita & 152 & FLSFNWGWLGVQQQRY-NWGPLTSNLLLVSQPGNI & DEQENFFAQASGFKRCMLFP \\
\hline C. intestinalis & 142 & FLQFNWDWVNKCQAAY-GWGSLSSNLLLIGLEGNI & DEQENFFAQISGHK \\
\hline N. vectensis & 149 & FLGFNWAWLNTMQKEM-DWGPLTSNLLLIGLPGNI & DEQQNLFCQVTGCKRVLLFH \\
\hline A. millepora & 149 & FLEFKWQWLTEMQRKM-NWGSLTSNLLLIGLPGNI & DEQQNFFGQLTGFKRVILFH \\
\hline S. purpuratus & 155 & FVRFNWNWVTDYQKKN-SWGPLTSNLLLVSMEGNV & DEQENFFAQVKGYKRFIMFP \\
\hline O. bimaculoides & 137 & FVSFNWRWISMQQKEN-NWGPLSSNLLLVGMAGNI & DEQQNFFAQLRGVKRCLLFA \\
\hline D. citri & 149 & FLHFNWDWAKNKQKIH-GWGPLTSNLLLLNIAGNV & EQQNLFVGIRGFKRCILFP \\
\hline T. cancriformis & 144 & YVKFNWPWIVNIQKRN-GWGSLTSNLLLISQGGNI & DEQHNLFAQIRGYKRFIFFP \\
\hline C. teleta & 142 & FLQFNWDFAKEQQKRN-GWGPLTSNLLLIGMAGNV & DEQENLFAQVRGYKRVILFP \\
\hline A. pisum & 137 & FVMFNWDWIMSKQKVC-KWGSLTSNLLLIAQEGNVT & DEQQNMFASIRGYKRFILFP \\
\hline H. sapiens & 162 & FLGFNWNWINKQQGKR-GWGQLTSNLLLIGMEGNVT & DEQQNFFAQIKGYKRCILFP \\
\hline B. floridae & 141 & FLQFNWDWINKQQQKQ-NWGHLTSNLLLIGMAGNVT & EQQNFFAQVKGFKRCILFA \\
\hline F. occidentalis & 154 & FIRFRWDWVTAKQKLH-NWGKLTYNLLLVGLEGNVT & EQENLFAQIHGHKRCILFP \\
\hline C. aquilonaris & 140 & FLGFNWPWINGQQKKH－GWGPLSSNLLLVGLGGNVT & EQENFFAQVRGHKRCILFP \\
\hline I. scapularis & 146 & FLRFNWDWVNRQQKHN-GWGPLTSNLLLVGTGGNVT & EQQNFFAHLRGHKRFLLFS \\
\hline A. geniculata & 137 & FLDFNWKWICEIQKQN-NWGPLTSNLLLVSSAGNVT & EQQNIFAQIRGFKRFILFP \\
\hline C. sculpturatus & 137 & FLDFNWNWISGQQQQH-NWGPLTSNLLLIGSEGSVT & EQQNFFAQIYGFKRFILFC \\
\hline S. olivacea & 150 & FLQFNWQWLNMQQKNN-NWGPLTSNLLLVGMEGNVT & EQQNFFSQLVGYKRCILFA \\
\hline T. castaneum & 148 & FVKFDWDYVNGKQTKH-NWGPLTSNLLFIAMEGNQ & DEQENFFAQVQGYKRCILFP \\
\hline S. maritima & 141 & FVRFNWNWANGRQKKN-NWGSLTSNLLLIATEGNITPC & YDEQQNFFAQVRGSKRVFLFP \\
\hline E. danica & 150 & FRNFNWDWVNNLQKKH-TWGPLTSNLMLIAMEGNVTPC & YDEQQNIFAQVYGYKRCILFH \\
\hline R. prolixus & 144 & FLGEKWDWINGIQKKM-NWGPLTSNLLLIGMEGNVTPC & YDEQENLFAQVRGYKRI I LFP \\
\hline P. humanus & 147 & FLHFKWDWLSNIQKLS-NWGPLTSNLLLISMEGNVTPC & DEQQNLFAQITGYKRCILFP \\
\hline C. pallens & 151 & FRGFNWSWVNVKQKAN-SWGPLTSNLLLISMEGNVTPC & YDEQQNFFAEVRGHKRCILFP \\
\hline Z. nevadensis & 145 & FLNFRWEWINSKQKMH-SWGPLTSNLLLIAMEGNVTPC & YDEQQNFFAEVRGFKRCILFP \\
\hline T. commodus & 170 & FLNFRWEWINAKQKAN-NWGPLTSNLLLISMEGNVTPC & IDEQQNFFAEVRGYKRCILFP \\
\hline
\end{tabular}


P. brassicae 179 PDGIPDAYMHPVGHARMGLVSDDDRQSQINDICHPDLAAFPAFAGLRGTQGVVEPGQALV

E. huxleyi 515 FVEYEKLYPFPVTHP------CDRQSMV-DVARPDLEAFPAFATASGQYTTLQPADLVH

B. natans 295 PGDWQGMYAFPCNHA-------CDRQAMV-NPDNPDLHRFPLYRHARPHYAVLQPGDLLY

191 PAEWGMYPFPLHHA-----CDROSMM-P--SHDRMLFPNFDRASGYFA IVHPGEMIYY

Chrysochromulina 378 PADFGCLYPFPMHHP-------CDRQSQV-DLYAPDLSRFPRFRAARPLEAILQPGEVLY

C. owczarzaki 284 PDRFPCLYPYPVHHP-------CDRQSQV-DFDNPDLARFPRFSELHGWECILEPGEVLY

O. cincta 249 PENEPYLYPYPVHHP-------NDRQSQV-NLDNPDYGRFPKFAMARGVQATVGPGEILY

S. rosetta 195 PDNERCLYPYRYGHP-------CDRQSQV-DFDNPDYERFPKFKDARGLEAILRPGDVLY

L. anatina 215 PSQFECLYPYPVYHP-------CDRQCQV-DLRAPDFDRFPKLKRLKGYEVIVKPGDVLY

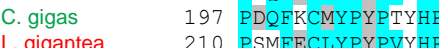

L. gigantea 210 PSMFECLYPYPVYHP------

A. queenslandica 203 PDQYDKLYPYPVAHP-------CDRQSQV-DFDSPDFERFPKFKEIEGYECILSPGDVLY

A. aurita 211 PSOFKCFYPFPYHHP-1

201 PDQEDCLYPHPVAHP-----CDR

N. vectensis

$\begin{array}{lll}\text { A. millepora } & 208 & \text { PDKFKCLYPFPVHHP } \\ \text { A. } & 208 \text { PDQFKCLYPFPLYHP }\end{array}$

S. purpuratus 214 PSOFDRTYPFPVH

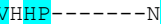

O. bimaculoides 196 PDQFKSLYPFPVYHP-------HDRS

$\begin{array}{ll}208 & \text { PEQFDCLYPPPVSHP------ } \\ \text { T. cancriformis } & 203 \text { PEQFENLYPHPFPHP-------HD }\end{array}$

C. teleta 201 PEOFSCLYPYPVHHP

A. pisum 196 PSEFECLYPHPVHHP

H. sapiens 221 PDQFECLYPYPVHHP

B. floridae 200 PEQIECLYPYPVHHP

F. occidentalis 213 PEQFGCLYPHPVWHP-

C. aquilonaris 199 PEQFDCLYPHPVYHP

l. scapularis 205 PDQYGCLYPHPVWHP

A. geniculata 196 PEQYECFYPYPVFHP--

C. sculpturatus 196 PEMYECLYPHPVWHP------ H

209 PEHYERLYPYPVYHP

T. castaneum 207 PSQFECLYPYPVHHP

S. maritima

E. danica

209
RDQFDCLYPHPIFHP

P. humanus 206 PEQFECLYPHPVYHP

C. pallens $\quad 210$ PEQFECLYPYPVYHP

Z. nevadensis 204 PEQFECFYPHPVYHP-

T. commodus

229 PEQFECFYPHPVYHP------HDRQSQV-DFDRPDYTRFPKFREARGQEAVVGPGDVLY

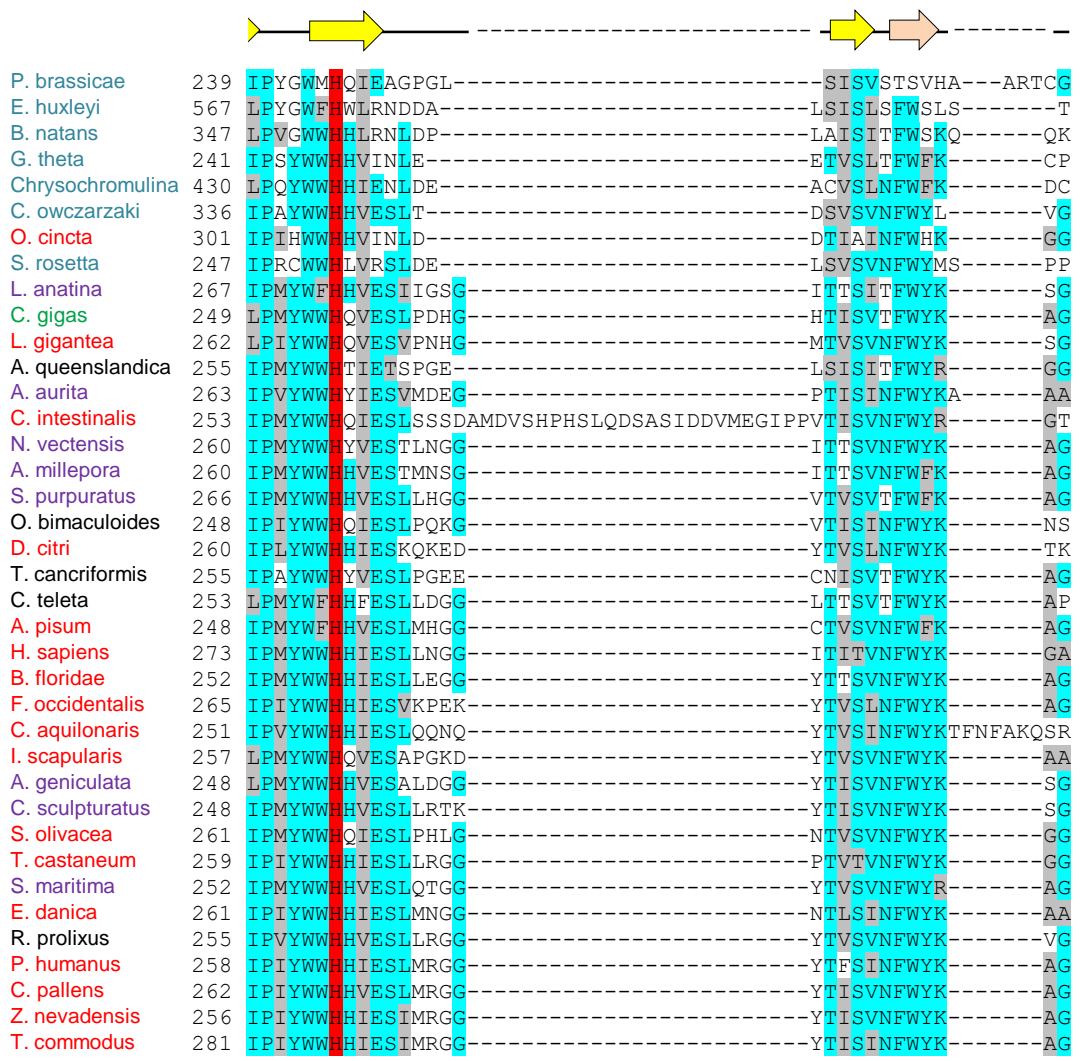


P. brassicae

E. huxleyi

B. natans

G theta

Chrysochromulina

C. owczarzak

O. cincta

S. rosetta

anatir

C. gigas

L. gigantea

A. queenslandica

A. aurita

C. intestinalis

N. vectensis

A. millepora

S. purpuratus

O. bimaculoides

D. citri

T. cancriformis

C. teleta

A. pisum

H. sapiens

B. floridae

F. occidentalis

C. aquilonaris

I. scapularis

A. geniculata

C. sculpturatus

S. olivacea

T. castaneum

S. maritima

E. danica

R. prolixus

P. humanus

C. pallens

Z. nevadensis

$\mathrm{T}$. commodus

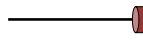

269 LPP-------AA--CAGIAGNLL---HDAPSAVRDI-----YLR

593 K-KERVPDVFSAHDLTLVRRNLEKHMAARFGAALFPORMRRIIRLI 374 ASLDTLKLPLDQRHLLTVRRNLEEMVFNIFGSESLQN-LAKOLSAKH-SSKPSTKISEG

456 AKPEKVVLPLSASQHLAMRRNIEKLVAQKLGATKAQL-VLPLLASHTDAEQPAESAMPE 361 PP--EIAHPLTYQQKISVMRNIEKMLGEALGDPTQL---SSLIS---------------

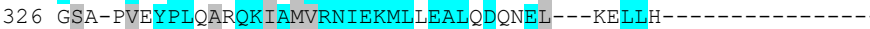
274 AIAEDIRFPLTASOKVAMMRNIEOMLLTALGSAGEV---SDFLR---------------

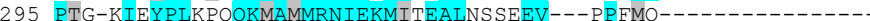
277 PIG-NVVYPLSPQQKVSMMRNLEKMIHQALNNTDEI---PEFMQ--------------290 TEE-KIVYPLKPQQKVAMMRNIEKMVTSAINNHEEV---PHLLK--

282 PVPSKITYPLSSQQKVAITRNIEKMLHEALQDHNEV---GPLLH-

306 PLPDVIQYPLSPQQRVSVMRNVEKMLAAALGDAEKV---GELLR--------------288 QTPSEISFPLSSQQKIAIMRNIERMLGDALGSHTEV---GPLIN-------------288 PTPSQITHPLTAQQKVAVMRNIERMLGEALGDPHEV---GPLLN

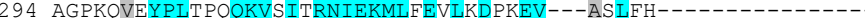

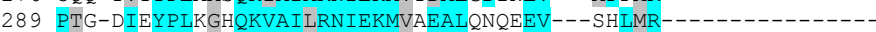
283 PTG-VVKYPLKGYQKVAIMRNIEKILVEALQDPQEV---GPLLR 281 PVG-KVEYPLKPQQKVAMMRN IEKMITEALNDQHEV---APFMR--_-_-_-_-_-_276 SVE-KIEYPITDHQKMVTMRNVEKMLATAL

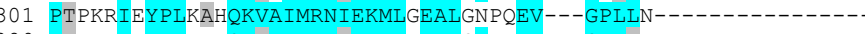
280 PTPRNIVYPLKPVQKMAIMRNLEKMLAEALGDYREV---GPLIN--------------293 PVG-QVEYPIKGYQKVAIMRNVEKMLAEAMODPEEV---GALIR-

286 PTG-DTVYPI

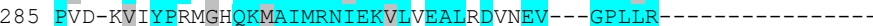
276 PTG-KVKYPLQASQKMAIMRNVEKMLVEALKDVNEV---GPLLR---------------276 PTG-KIKYPLNGHQKMAIMRNIEKMLVEALKDVNEV---GPLLR--------------289 PTE-KIEYPLKPRQKLAIMRNVEKMLLEALREPAEV---GPLLR---------------

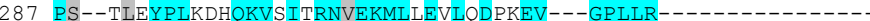
280 PTG-KIEYPLKAQQKVAI IRNVEKMVSGALEKPEEV---GPLLR--------------289 PPP-PISYPLLGHQKIAIMRNVEKMLAEALQDQKEV---GPLLR--------------283 PTG-HITYPLKGYQKVAIMRNIEKMLVEALKDTEEV---GPLLR---------------

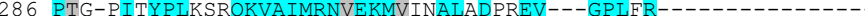
290 PTG-EIVYPLKGHOKVAIMRNVEKMLLEALODPREV---GPLLR---.--- - - - - - 284 PTG-QIVYPLKDHQKVAIMRNVEKMLVEALQDPHEV---GPLLR------------

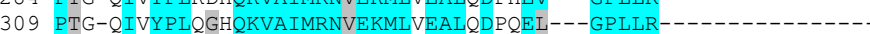

\begin{tabular}{|c|c|c|}
\hline & & \\
\hline & 650 & VGEVLAEARTLLGAVQVADPEKQDEFLRSMLRVRFEG \\
\hline & & NSKVKE FMVNCLSNF--MGRTQAEPLI LDMLEGRYCN \\
\hline & 6 & - - - - - - - - - - - \\
\hline chromulina & 14 & VGALREEVTRLLTHV--MPAADVPAWLCELVEGRFPF \\
\hline & & ------------------------- DLVLGRYTH \\
\hline & & 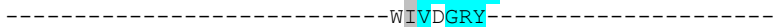 \\
\hline & & $---------------------------\mathrm{ML}$ \\
\hline & & $--------------------------\mathrm{NM}$ \\
\hline & & $---------------------------\mathrm{NM}$ \\
\hline & & ---------------------------TMVL \\
\hline lica & & $--------------------------M I C D G$ \\
\hline & & ---------------------------- MIVNG \\
\hline & & $-----------------------------\mathrm{TM}$ \\
\hline & & $----------------------------\operatorname{LN}$ \\
\hline & & $---------------------------\mathrm{CM}$ \\
\hline & & 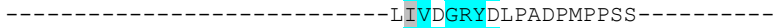 \\
\hline & & $---------------------------\mathrm{TM} \mathrm{T}$ \\
\hline & & 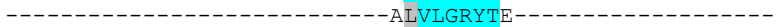 \\
\hline & & $----------1--1-------------\mathrm{AM}$ \\
\hline & & 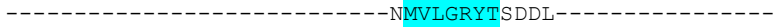 \\
\hline & & 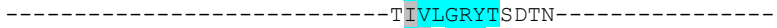 \\
\hline & & $---------------------------\mathrm{T}$ \\
\hline & & $-------------------------\mathrm{TM}_{\mathrm{I}}$ \\
\hline & & 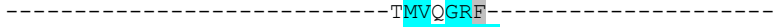 \\
\hline & & ---------------------------- TMT \\
\hline & & 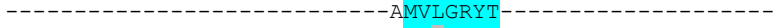 \\
\hline & & ---------------------------- SMVI GRYT \\
\hline turatus & & 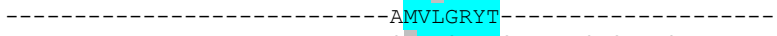 \\
\hline & & 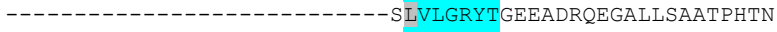 \\
\hline & & ---------------------------SMVLGRYTE---------------- \\
\hline & & 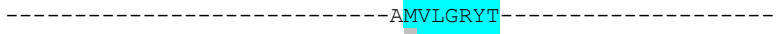 \\
\hline & & 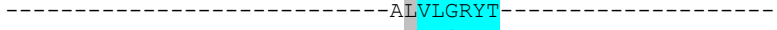 \\
\hline & & ------------------------ AIVLGR \\
\hline & & -------------------------- ALVLGF \\
\hline & & ---------------------------- TLVLG \\
\hline & & -------------------------- ALVLG \\
\hline & & \\
\hline
\end{tabular}

